\title{
Comparative Study between Treatment by Vacuum-Assisted Closure (VAC), Treatment by Hyperbaric Oxygen Therapy and Traditional Treatment of Diabetic Foot Infection
}

\author{
Ahmed El-Said Mousa*, Mahmoud Salah Shehata, Mohamed Mohsen Salem \\ Department of General Surgery, Faculty of Medicine, Al-Azhar University, Cairo, Egypt. \\ * Corresponding author: Ahmed El-Said Mousa Ibrahim, email: dr_wiseman@yahoo.com
}

\begin{abstract}
Aim: To compare the therapeutic effects of vacuum-assisted closure therapy, hyperbaric oxygen therapy, and traditional moist dressings in improving the healing process of chronic diabetic foot infection.

Study design: A prospective randomized study.

Patients and Methods: A total of 75 patients, with a chronic diabetic foot infection, were included in this study. After surgical debridement, all patients were randomly classified into 3 groups, with 25 patients in each group. Group A were treated by VAC therapy, Group B were treated by HBOT and Group C were treated by traditional moist dressings respectively. Wounds were evaluated every week for six weeks as regards the size, timing of healthy granulation tissue formation, local wound complications, and number of debridement sessions.

Results: There was a significant reduction in the wound size in group A with a mean of $70.25 \%$, B with $62.65 \%$ compared to group $\mathrm{C}$ with $32.46 \%$. As regards healthy granulation tissue formation onset, it was more rapid in group A with a mean of 6.5 , B with 7 compared to group $\mathrm{C}$ with 14 days. As regards the number of debridement sessions and local wound complications they were significantly less in group A than the other two groups.

Conclusion: Vacuum-assisted closure (VAC) therapy appears to be safe, more effective with less complications than the other two methods in the treatment of diabetic foot infection, and can be used with HBOT simultaneously if indicated.
\end{abstract}

Keywords: Vacuum-assisted closure, VAC, hyperbaric oxygen therapy, traditional moist dressings, chronic diabetic foot infection.

\section{INTRODUCTION}

The management of diabetic foot ulcers (DFU) has become a major public health concern as the incidence of diabetes continues to increase. Diabetes mellitus is widespread as regard the Egyptian society nowadays, nearly $10.4 \%$ of the Egyptian population has Diabetes mellitus ${ }^{[1]}$.

The standard care for diabetic foot ulcers includes; blood glucose level control, treatment of infection, adequate and frequent debridement followed by moist dressings, revascularization in the case of the ischemic limb ${ }^{[2]}$. Only $30 \%$ of DFUs heal within 12 weeks even after recent advances in traditional moist dressings and so increases the risk for serious complications such as hospitalization, infection, and the possibility of amputation ${ }^{[3]}$.

Traditional treatment alone may not be enough to heal diabetic foot infection and adjunctive methods may be necessary. Two different modalities have been suggested as effective methods; vacuum-assisted closure (VAC) therapy, and hyperbaric oxygen therapy (HBOT) ${ }^{[4]}$.

Hyperbaric oxygen therapy involves the inhalation of $100 \%$ oxygen at pressures 2 or 3 times greater than atmospheric pressure at sea level. This method is used primarily to aid the healing of diabetic foot ulcers and proved to be effective in decreasing major amputation in diabetic patients with resistant foot ulcers ${ }^{[5]}$.
Topical negative pressure application is said to increase local blood flow to the tissues, reduces edema, and decrease bacterial colonization and infection rate. It is thought to promote wound healing by accelerating the granulation tissue formation as well as by exerting mechanical effects on the wound. It also provides an ideal wound healing environment by keeping a moist environment and removes excess wound exudates ${ }^{[6]}$.

The present study was conducted to compare the efficacy of vacuum-assisted closure therapy, hyperbaric oxygen therapy, and traditional wound dressings in improving the healing process and reducing the complication rate in chronic diabetic foot infection.

\section{Ethical Considerations:}

This study was carried after obtaining approval from the local ethical committee of the faculty of medicine, Cairo, Al-Azhar university, and after fully informed written consent signed by every patient was obtained.

\section{PATIENTS AND METHODS}

A prospective randomized study was carried out in the diabetic foot department at Raas Al-teen General Hospital. A total of 75 patients, with chronic diabetic foot ulcers were included in this study. After surgical debridement, all patients were randomly classified into 
3 groups, with 25 patients in each group. Patients in Group A were treated by vacuum-assisted closure therapy, patients in Group B were treated by hyperbaric oxygen therapy and patients in Group C were treated by traditional moist dressings respectively.

\section{Exclusion criteria}

1) Patients with acute ischemia

2) Patients with a completely neglected limb or with gangrene

3) Patients with osteomyelitis or Charcot joint

4) Patients with exposed arteries or veins

5) Patients who cannot tolerate hyperbaric oxygen therapy as COPD.

\section{All patients were subjected to:}

1- Detailed history including; personal data, wound onset and duration, previous surgical intervention, diabetes mellitus onset and control, presence of other medical conditions, smoking.

2- Clinical examination;

- Detailed general examination.

- Local examination;

1- Wound examination: site, size, number, shape, discharge.

2- Vascular examination: pulse, trophic changes.

3- Neurological examination: paresthesia, sensory loss.

3- Investigations:

- Laboratory; complete blood count, fasting and postprandial blood glucose, and glycosylated hemoglobin level $\left(\mathrm{HbA}_{1} \mathrm{C}\right)$, culture, and sensitivity test from the wound discharge.

- Imaging studies: A colored duplex study of both lower limbs and Plain x-ray film (anteroposterior, lateral, oblique) were done to exclude osteomyelitis of the affected foot.

\section{Materials:}

- Group (A) vacuum-assisted closure therapy VAC:

1- Vacuum suction apparatus; automatic vacuum pump (negative pressure unit), canister $(500 \mathrm{ml}$ capacity), tubing to connect the dressing to the pump.

2- Synthetic hydrocolloid sheet

3- Transparent adhesive membrane sheet

4- Fenestrated evacuation tube (Redivac 18)

- Group (B) hyperbaric oxygen therapy:

1- The hyperbaric oxygen therapy unit

- Group (C) Traditional moist dressing:

1- Normal saline

2- Povidone-iodine solution $10 \%$

3- Topical healing stimulator cream (NATARIA Cream ${ }^{\mathrm{R})}$

4- Sterile gauze

5- Adhesive tapes

\section{Technique:}

All wounds were adequately surgically debrided, adequate hemostasis was obtained and various wound dimensions were measured.

- Group (A): The first step is to prepare the wound area by removing any old dressings and obtain normal dry surrounding healthy skin. If required, a swab for microbiology should be taken.

A synthetic hydrocolloid sheet that is remodeled to fill the wound cavity after being soaked in povidoneiodine is placed. A Fenestrated tube (Redivac 18) was placed over the previous sheet and the distal end was brought out to a dry healthy skin area away from the wound.

The whole area was wrapped with a transparent adhesive sheet to be both air and fluid sealed. The automatic vacuum pump was connected to the other end of the tube drain and negative pressure of -125 $\mathrm{mmHg}$ was created in an automatic intermittent manner (30 minutes on, 10 minutes off). The success of the connection is proved by the shrunk of the hydrocolloid sheet after the device turned on so, the discharge is absorbed by the foam and removed by the apparatus. The dressing was changed, and the wound is inspected and debrided after 1 week except if there was a need to change the dressing earlier.

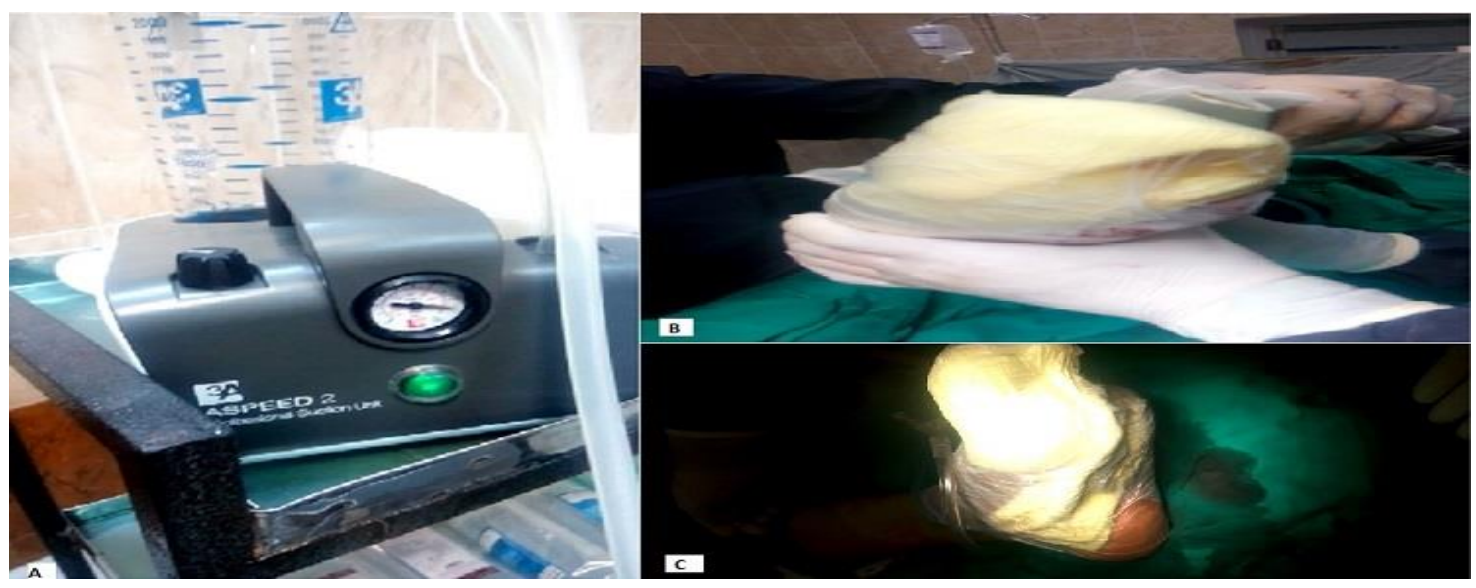

Fig-1: A; vacuum suction apparatus - B; the shape of the wound while suction is off-C; the shape of the wound while suction is on. 
- Group (B): Hyperbaric oxygen therapy was used in these patients in the following manner; Each patient had a hyperbaric oxygen therapy session once daily for the first 15 days and then had rest for the next 10 days followed by another 15 sessions in the last 15 days.

- Group (C): The wound at first was irrigated with normal saline and then soaked with povidone-iodine solution $10 \%$. The local application of healing

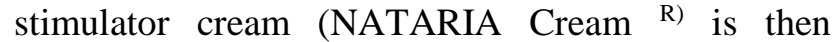
applied. The dressing was done two or three times daily according to wound condition. Surgical debridement was done when indicated.

\section{Follow-up:}

Diabetic foot wounds were evaluated every week for six weeks. Follow up were conducted through clinical evaluation of the wound according to the following:

1- Reduction in the wound size

2- The time needed for healthy granulation tissue formation in days

3- Number of surgical debridement sessions

4- Local wound complications (cellulitissecondary amputation).

Definitive wound closure by skin grafting was suitable in cases of wounds that showed incomplete healing but had healthy granulation tissue during the follow-up period.

\section{RESULTS AND STATISTICAL ANALYSIS}

I. Personal Data: The studied population included 48 females and 27 males. Their ages ranged between 35 and 72 years with a mean of 52.46 years (Table 1).

Table 1: Comparison of gender and age distribution

\begin{tabular}{|c|c|c|c|}
\hline Personal Data & VAC & HBOT & Traditional \\
\hline \multicolumn{4}{|l|}{ 1- Gender } \\
\hline Male & $9(12 \%)$ & $11(14 \%)$ & $7(10 \%)$ \\
\hline Female & $19(25 \%)$ & $13(18 \%)$ & $16(21 \%)$ \\
\hline \multicolumn{4}{|l|}{ 2- Age (years) } \\
\hline Min. - Max. & $38-72$ & $35-65$ & $37-61$ \\
\hline Mean & 53.28 & 49.36 & 55.43 \\
\hline
\end{tabular}

II. Local wound examination (before treatment): The clinical wound examination findings before the beginning of treatment in all groups are summarized in (Table 2).

Table 2: Comparison of local wound examination findings before treatment

\begin{tabular}{|c|c|c|c|}
\hline $\begin{array}{c}\text { Wound Findings } \\
\text { 1- Duration (months) } \\
\text { Min. - Max. }\end{array}$ & VAC & HBOT & Traditional \\
\hline $\begin{array}{c}\text { Mean } \\
\text { 2- Largest dimension }\end{array}$ & $1.75-3.5$ & $2.25-3.85$ & $1.25-4.0$ \\
\hline Min. - Max. & 2.25 & 3.00 & 2.25 \\
\hline Mean & $6.5-11$ & $5.0-11.5$ & $4.5-10$ \\
\hline 3- Site & 8.5 & 8 & 7.5 \\
\hline Front & 7 & 9 & 7 \\
\hline Med. Sole & 5 & 3 & 2 \\
\hline Lat. Sole & 8 & 6 & 9 \\
\hline Heel & 5 & 7 & 7 \\
\hline
\end{tabular}

III. Local wound examination (after six weeks): The clinical wound examination findings after six weeks of treatment in all groups are summarized in (Table 3).

Table 3: Comparison of the wound after six weeks of treatment

\begin{tabular}{|c|c|c|c|}
\hline $\begin{array}{c}\text { Follow-Up } \\
\text { (After six weeks) }\end{array}$ & VAC & HBOT & Traditional \\
\hline $\begin{array}{c}\text { 1- Size (cm) } \\
\text { Min. - Max. } \\
\text { Mean }\end{array}$ & $2-3.5$ & $2-4.5$ & $3-6.5$ \\
\hline 2- Percentage of size reduction (\%) & 2.5 & 3 & 5 \\
\hline
\end{tabular}

The previous table shows that there was a significant reduction in the size of the wound in both groups A and B compared to group $\mathrm{C}$ after six weeks of treatment. 
IV. Follow up data: Comparison between the three groups as regards healthy granulation tissue formation onset, the number of debridement sessions, and local wound complications are summarized in (Table 4).

Table 4: Comparison of follow up clinical data after six weeks of treatment

\begin{tabular}{|c|c|c|c|}
\hline Follow-Up (After six weeks) & VAC & HBOT & Traditional \\
\hline \multicolumn{4}{|l|}{ 1- Healthy granulation tissue formation (days) } \\
\hline Min. - Max. & $4-12$ & $6-14$ & $7-21$ \\
\hline Mean & 6.5 & 7 & 14 \\
\hline \multicolumn{4}{|l|}{ 2- Number of debridement sessions } \\
\hline Min. - Max. & $1-3$ & $1-4$ & $3-8$ \\
\hline Mean & 1.5 & 2 & 5 \\
\hline \multicolumn{4}{|l|}{ 3- Wound infection } \\
\hline & $2(8 \%)$ & $5(20 \%)$ & $15(60 \%)$ \\
\hline \multicolumn{4}{|l|}{ 4- $2^{\text {nd }}$ amputation } \\
\hline & 0 & 0 & $2(8 \%)$ \\
\hline
\end{tabular}

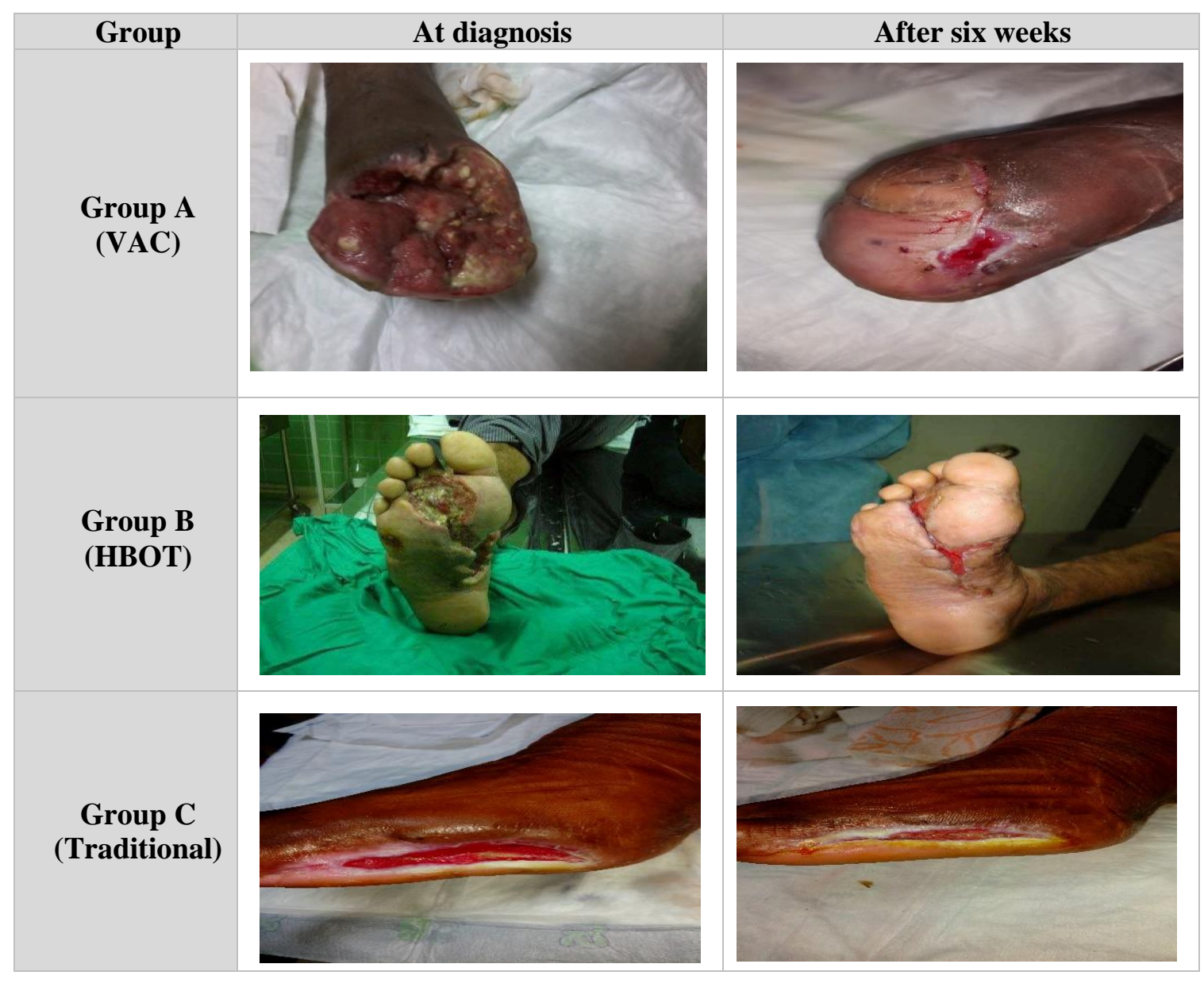

Table 5: Clinical comparison before and after six weeks of treatment

\section{DISCUSSION}

The prevalence of diabetic foot ulcers is increasing by $9 \%$ annually. Diabetic foot ulcers are difficult to treat due to the presence of factors such as infection, angiopathy, and neuropathy that prevent the natural wound healing process and generally diabetic wounds take a longer time to heal and care is enormously variable ${ }^{[7]}$.
Despite great advances in different medical specialties, the management of chronic wounds remains a tough challenge, and a lot of dressing modalities, local applicants, and a lot of studies are still going on. Chronic wounds are usually treated sub-optimally with general wound care products designed simply to cover and absorb exudate. Despite the long history of use of different wound dressing varieties, there is no consensus about the ideal dressing and so surgical 
dressing preference is based mainly on tradition and the surgeon own experience ${ }^{[8]}$.

The application of local negative pressure with a closed system (vacuum) creates a hypoxic environment and removes the wound discharge and thus reduces infection rates and increases localized blood flow, thereby supplying the wound with oxygen and nutrition to accelerate wound healing ${ }^{[9]}$.

Hyperbaric oxygen therapy is achieved by having the patient breathing concentrated oxygen at a pressure higher than 1 absolute atmosphere. In ischemic tissue, increasing $\mathrm{pO} 2$ in the tissues improves the phagocytic function of the macrophages and the environment becomes less suitable for anaerobic bacteria. Also, it improves the transport of certain antibiotics across the bacterial cell wall ${ }^{[10]}$.

In the present study, we compare the efficacy of VAC, HBOT, and traditional moist wound dressings in improving the healing process and reducing the complication rate in chronic diabetic foot wounds.

In this study, VAC showed better results as regard reduction in the wound size $(70.25 \%)$ compared to $(62.65 \%) \&(32.46 \%)$ for HBOT and traditional dressing respectively. The onset of healthy granulation tissue formation occurred early within a mean of (6.5) days compared to (7) \& (14) days in HBOT and traditional dressing respectively. It was noted also that the mean of debridement sessions in the VAC group was (1.5) times compared to (2) \& (5) in HBOT and traditional dressing respectively. Moreover, the incidence of secondary infection and amputation were less in the VAC group compared to HBOT and traditional dressing treatment as shown in table 4.

We reviewed many studies that were consistent with those results; Joseph et al ${ }^{[9]}$. observed that after six weeks, chronic diabetic foot wounds treated with VAC had a significantly greater percent reduction in wound volume and depth than wounds treated with the traditional methods, Arti et al ${ }^{[11]}$. found that VAC therapy succeeded to achieve complete ulcer closure to a major extent greater than that for the traditional wound therapy group. In a controlled randomized trial, Armstrong et al ${ }^{[12]}$. have demonstrated that VAC therapy is a better option in treating outcomes of surgical wounds from minor amputations compared to traditional wound therapy.

As regards wound complications, wound infection and cellulitis develop more in traditional methods than VAC, corresponding to these results, Paola et al ${ }^{[13]}$. found that infection control was better and faster in the VAC group than in the conventional group.

\section{CONCLUSION}

This study showed that vacuum-assisted closure (VAC) appears to be safe and more effective than hyperbaric oxygen therapy (HBOT) and traditional methods for the treatment of diabetic foot ulcers; as VAC has better results in wound healing; it provides a significant reduction in the size and depth of the wounds, faster healthy granulation tissue formation, less number of debridement sessions and less incidence of local wound complications compared to the other two groups and also can be used with HBOT in the same patient simultaneously if indicated.

\section{REFERENCES}

1. Soliman AO (2013): Diabetes Mellitus in Egypt in Short. J Diabetes Metab., 4: 318. doi:10.4172/2155-6156.1000318.

2. Yazdanpanah L, Nasiri M, Adarvishi S (2015): Literature review on the management of diabetic foot ulcers. World Journal of Diabetes, 6(1): 37-53.

3. Zhang J, Hu Z, Chen D, Guo D, Zhu J, Tang B (2014): Effectiveness and safety of negative-pressure wound therapy for diabetic foot ulcers: a meta-analysis. Plastic and Reconstructive Surgery, 134(1): 141-151.

4. Blume P, Walters J, Payne W, Ayala J, Lantos J (2008): Comparison of negative wound therapy using vacuumassisted closure with advanced moist wound therapy in the treatment of diabetic foot ulcers: a multicenter randomized controlled trial. Diabetes Care, 31(4): 631-636.

5. Goldman R (2009): Hyperbaric oxygen therapy for wound healing and limb salvage: a systematic review. PMR., 1(5): 471-89.

6. Dang C, Boulton A (2003): Changing perspectives in diabetic foot ulcer management. Int $\mathrm{J}$ Lower Extremity Wounds, 2: 4-12.

7. Banwell P, Teot L (2003): Topical negative pressure (TNP): the evolution of a novel wound therapy. J Wound Care, 12: 22-8.

8. Vermeulen H, Goossens A, Vos R, Legemate D (2005): Systematic review of dressings and topical agents for surgical wounds healing by secondary intention. Br J Surg., 92 (6): 665- 672.

9. Joseph E, Hamori C, Bergman S, Roaf E, Swann N, Anastasi G (2000): A prospective randomized trial of vacuum-assisted closure versus standard therapy of chronic nonhealing wounds. Wounds, 12(3): 60-67.

10. Knighton D, Halliday B, Hunt T (1986): Oxygen as an antibiotic. A comparison of the effects of inspired oxygen concentration and antibiotic administration on in vivo bacterial clearance. Arch Surg., 121(2): 191-195.

11. Arti H, Khorami M, Ebrahimi V (2016): Comparison of negative pressure wound therapy (NPWT) \& conventional wound dressings in the open fracture wounds. Pak J Med Sci., 32(1): 65-9.

12. Armstrong D, Lavery L, Abu-Rumman P, Espensen E, Vazquez J, Nixon B, Boulton A (2002): Outcomes of subatmospheric pressure dressing therapy on wounds of the diabetic foot. Ostomy/Wound Management, 48(4): 64-8.

13. Paola L, Carone A, Ricci S, Russo A, Ceccacci T, Ninkovic S (2010): Use of Vacuum-Assisted Closure Therapy in the Treatment of Diabetic Foot Wounds. JDFC., (2): 33-44. 